\title{
Visualization of the shear wave on gray-scale ultrasound: experience with a complex breast cyst
}

\author{
Angelica Chiorean ${ }^{1,2}$, Roxana Pintican', Maria Magdalena Duma², Radu Fechete ${ }^{3}$, Diana \\ Feier', Madalina Szep², Sorin Dudea ${ }^{1}$
}

${ }^{1}$ Department of Radiology, "Iuliu Hatieganu” University of Medicine and Pharmacy, ${ }^{2}$ Medimages Breast Center, ${ }^{3}$ Department of Physics, Technical University, Cluj-Napoca, Romania

\begin{abstract}
Shear wave elastography (SWE) is the newer of the two methods of measuring tissue stiffness using ultrasound. We present the case of a 39-year-old patient with fibrocystic breast disease who presented with a left painful breast lump. The patient underwent a breast ultrasound that depicted a complicated cyst with a "pseudo-solid" content. When the SWE mode was turned on, the ultrasound share wave was observed on the gray-scale image, as surface undulations of the "pseudo-solid" component. To the best of our knowledge, this is the first report of the share wave visualization on a gray-scale image. The impact on patient management is discussed.
\end{abstract}

Keywords: shear wave; breast elastography; complicated cyst

\section{Introduction}

Share-wave elastography (SWE) is based on the principle of acoustic radiation force produced by the ultrasound (US) probe. Transversely oriented shear-waves are generated by the acoustic radiation force and used to induce tissue deformation [1]. SWE measures the shear-wave propagation speed and displays the quantitative data as a color-coded image in real time. Waves propagate faster in hard tissue than soft tissue and SWE is proved useful in improving the specificity of US evaluation of BI-RADS 3 and 4a breast lesions, including complicated cysts [2]. We report the shear-wave direct

Received 18.10.2019 Accepted 11.12.2019

Med Ultrason

2020:0 Online first, 1-3

Corresponding author: Roxana Pintican

2/1 Plopilor street,

400379, Cluj-Napoca, Romania

Phone: +40 729121.122

E-mail: roxana.pintican@gmail.com visualisation on a gray-scale image and discuss its possible implication.

\section{Case report}

A 39 year old female patient, with known fibrocystic breast disease presented with a left painful breast lump. The patient was examined using a Supersonic Imagine Aixplorer machine (Aix-en-Provence, France, 2019). Breast US revealed multiple cystic lesions with various sizes that were diffusely dispersed in the two breasts. Corresponding to the left painful breast lump, a complicated cyst was identified in the upper-outer quadrant of the breast. The cyst had an imperceptible wall and a lowlevel echogenic content that mimicked a "pseudo-solid" component. Flow was absent at color Doppler, suggesting that the cyst had an impure liquid content (sediment or debris) rather than being a proliferative process (fig 1).

Applying 2D-SWE on the complicated cyst, the expected colour void and soft appearance of the debris was observed. Furthermore, the debris displayed an uncom- 


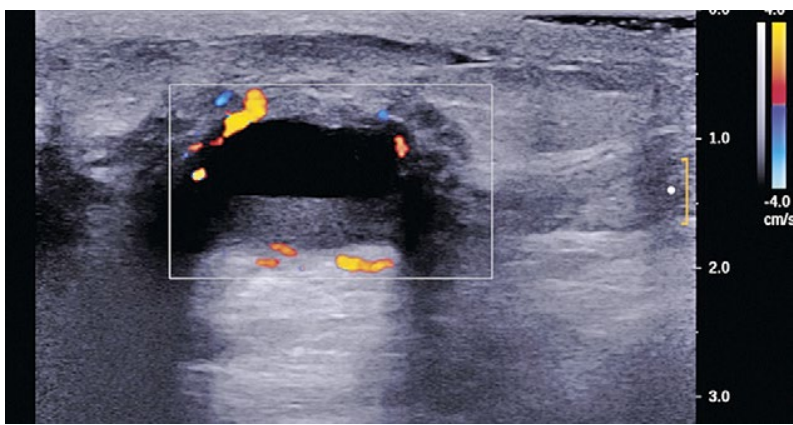

Fig 1. Complicated cyst without color Doppler flow in the "pseudo-solid" component.

mon gray-scale appearance with a rhythmical throbbing or vibrating movements, similar to an arterial pulsation (fig 2, supplementary video 1, on the journal site).

Both color and Power Doppler were still negative. The overall US findings were categorised as BIRADS 2. The patient underwent cyst aspiration and its entire content was completely evacuated (fig 3 ).

\section{Discussion}

Strain elastography was the first method used in clinical practice to assess the tissue stiffness. Several studies have already shown that is a useful tool in the diagnosis and characterization of breast lesions [1,3]. Furthermore, the vast user experience in strain elastography has lead to the discovery of numerous and well-described artifacts and pitfalls [4].

SWE, a relatively new elastography technique, is divided in two types: point shear-wave speed measurement and shear-wave speed imaging. Thereby, as new technologies emerge, new imaging findings, artifacts and pitfalls need to be reported as in the case of strain elastography.

A study reported that SWE improves the specificity of US evaluation of BI-RADS 3 and $4 \mathrm{a}$ lesions and included complicated cysts among examined lesions [2], but offered no other comments regarding share-wave imaging [2]. The latest WFUMB guidelines suggest that, in cystic lesions, SWE examination deserves a special attention as fluids do not support shear-waves and so cysts appear as colour voids [5]. When the fluid is more viscous, SWE may display soft regions.

Recent papers identify, describe and explain technical restrictions and artifacts encountered in 2D-SWE that may alter the appropriate diagnostic and therapeutic patient management $[5,6]$. Few of them are found only on Supersonic machine $[7,8]$.

Our case highlighted a new, to the best of our knowledge, unreported SWE imaging aspect, due to an expected physic phenomenon. The peculiar gray-scale appearance might be explained by the wave interference phenomenon, by which two waves superpose, forming a resultant wave of higher, lower, or equal amplitude. In our case, this translates into a rhythmical pulsation of the medium. Nothing but the debris was responsible for causing the phenomenon, as in pure transonic or completely impure cysts, the phenomenon was not seen.

For an unexperienced breast radiologist, this SWE appearance could erroneously indicate a vascularized mass. When using Supersonic SWE to evaluate the stiffness of a supposably intracystic mass, the users need to be aware of this normal phenomenon and must not rec-

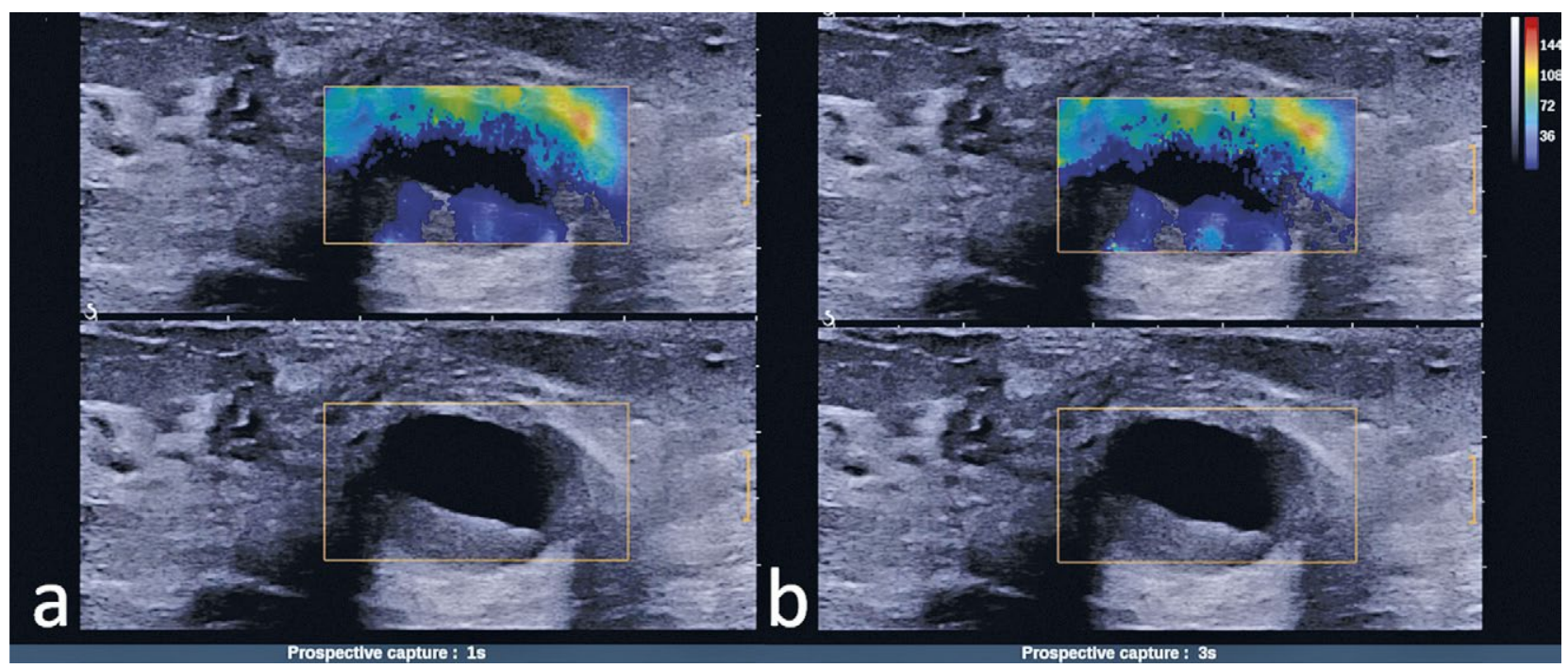

Fig 2. The cyst's debris displays rhythmical throbbing on gray-scale image, seen only when 2D SWE mode is on: high level at 1s (a) respectively low level at $3 \mathrm{~s}$ (b). 


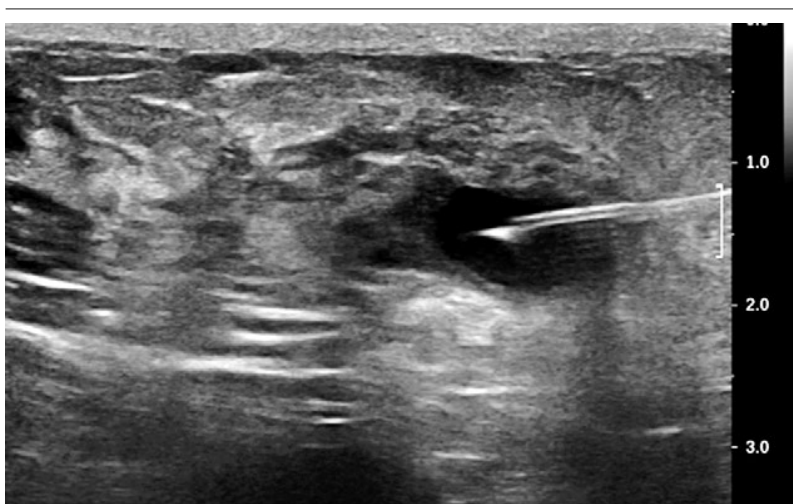

Fig 3. The cyst's fine needle aspiration: the cyst's wall collapsed and the entire content (debris and pure liquid) was evacuated.

ommend a biopsy, unless the lesion is investigated by other complementary ultrasound modes (2D gray-scale, color or cower Doppler) and is categorized as suspect (BIRADS 4 or 5 ).

In conclusion we present the first gray-scale image of shear-wave visualization together with its important clinical implications. As a future direction, it remains to be established if the share-wave visualization is linked only to Supersonic or it may be observed using other manufacturers' machines.

\section{References}

1. Hooley RJ, Scoutt LM, Philpotts LE. Breast ultrasonography: state of the art. Radiology 2013;268:642-659.

2. Berg WA, Cosgrove DO, Doré CJ, et al. Shear-wave elastography improves the specificity of breast US: the BE1 multinational study of 939 masses. Radiology 2012;262:435449.

3. Chiorean A, Duma M, Dudea S, et al. Real-time ultrasound elastography of the breast: state of the art. Med Ultrason 2008; 10:73-82.

4. Ciurea A, Dumitriu D, Ciortea C, Botar-Jid C, Dudea SM. Artifacts and pitfalls in breast elastoultrasonography: a pictorial essay. Med Ultrason 2008;10:93-98.

5. Shiina T, Nightingale KR, Palmeri ML, et al. WFUMB guidelines and recommendations for clinical use of ultrasound elastography: Part 1: basic principles and terminology. Ultrasound Med Biol 2015;41:1126-1147.

6. Bouchet P, Gennisson JL, Podda A, Alilet M, Carrié M, Aubry S. Artifacts and Technical Restrictions in 2D Shear Wave Elastography. Ultraschall Med 2018 Dec 21. doi:10.1055/a-0805-1099.

7. Bercoff J, Tanter M, Fink M. Supersonic shear imaging: a new technique for soft tissue elasticity mapping. IEEE Trans Ultrason Ferroelectr Freq Control 2004;51:396-409.

8. Lin CY, Chen PY, Shau YW, Wang CL. An Artifact in Supersonic Shear Wave Elastography. Ultrasound Med Biol 2017;43:517-530. 